\title{
Latent inhibition in flavor-preference conditioning: Effects of motivational state and the nature of the reinforcer
}

\author{
Felisa González $^{1,5}$ • Enrique Morillas ${ }^{2,5}$ - Geoffrey Hall ${ }^{3,4}$
}

Published online: 16 June 2015

(C) Psychonomic Society, Inc. 2015

\begin{abstract}
In two experiments, rats received pairings of the flavor of almond with either fructose or maltodextrin, and the conditioned preference for almond was then tested. In each experiment, half of the rats had received prior exposure to almond on its own, and half had received no preexposure. In Experiment 1, in which the rats were hungry during the test, the preference was greater in the nonpreexposed subjects, both for those trained with fructose and those trained with maltodextrin; that is, latent inhibition was obtained with both reinforcers. In Experiment 2, in which the rats were not food deprived prior to the test, not only was there no latent inhibition with either of the reinforcers, but, for both, the preference was greater for preexposed than for nonpreexposed subjects. These results give no support to the proposal that different types of reinforcer generate different types of learning. They are, however, consistent with the proposal that different types of learning control behavior when a rat is hungry and when it is not, and that the form that generates the preference in the latter case is not susceptible to the latent inhibition effect.
\end{abstract}

Felisa González

fgreyes@ugr.es

1 Mind, Brain, and Behavior Research Center (CIMCYC), University of Granada, Granada, Spain

2 Institute of Neurosciences, Centre for Biomedical Research (CIBM), University of Granada, Granada, Spain

3 Department of Psychology, University of York, York, UK

4 School of Psychology, University of New South Wales, Sydney, NSW, Australia

5 Department of Experimental Psychology, University of Granada, Campus Cartuja, 18071 Granada, Spain
Keywords Latent inhibition · Flavor preference · Fructose · Maltodextrin $\cdot$ Conditioning $\cdot$ Rats

Prior exposure to the event to be used as the conditioned stimulus (CS) in classical conditioning is usually found to retard acquisition of the conditioned response. This latent inhibition effect is robust and is readily obtained in a wide variety of conditioning procedures (see Lubow, 1989). An exception, however, is the flavor-preference conditioning procedure used in the experiments considered here. In this procedure, subjects (rats, in these experiments) are allowed to consume a neutral or nonpreferred flavor that is presented in compound with a substance of positive motivational value (such as a sucrose solution). After this training, rats given a choice between plain water and water containing the flavor show an increased tendency to consume the latter, an outcome that has been interpreted as an instance of conditioning, with the flavor serving as the CS and sucrose as the unconditioned stimulus (US). Exposure to the CS prior to such conditioning has produced varying results. De la Casa, Márquez, and Lubow (2009) found a reduction in the preference (i.e., latent inhibition), whereas Delamater (2011) found no effect (at least on initial testing; a difference emerged on a further test given after the rats had been given exposure to the US alone).

One factor that contributes to the outcome in this procedure appears to be the motivational state of the rat. In a series of experiments by Garcia-Burgos, González, and Hall (2013), rats were given preexposure to a solution of almond essence prior to pairing almond with sucrose. ${ }^{1}$ In all of these experiments, the rats were water deprived throughout the procedure

\footnotetext{
${ }^{1}$ Almond is presumed to function principally as an odor, but since it may also have a taste component, we will refer to it as a flavor.
} 
in order to ensure that the stimuli, presented as fluids, would be consumed readily. In some experiments, they were also made hungry throughout the experiment or prior to the test, and in these the latent inhibition effect was evident. We note that in the experiments by De la Casa et al. (2009), in which latent inhibition was obtained, the rats were food deprived throughout. In the study by Delamater (2011) the rats were not food deprived, and Garcia-Burgos et al. found no latent inhibition when the animals were not food deprived before the test. In these circumstances a sizeable preference was still found, but this was as large (in fact, slightly larger) in subjects given preexposure to the CS flavor as in subjects not given preexposure. The absence of latent inhibition after CS preexposure is surprising and interesting, and the aims of the present study were to attempt to replicate this finding under different conditions and to investigate the processes that might underlie it.

A possible interpretation of these findings has come from the suggestion that more than one mechanism can contribute to the preference established with sucrose as the US. Sucrose has both a sweet taste and nutritive postoral consequences, and each of these properties is capable of supporting preference conditioning. A palatable but nonnutritive substance (such as saccharin) will serve as an effective US (e.g., Fanselow \& Birk, 1982), a phenomenon referred to as flavor-taste learning. But a preference can also be formed when a nutrient US is delivered by intragastric infusion (e.g., Sclafani, Cardieri, Tucker, Blusk, \& Ackroff, 1993), so that its taste properties are irrelevant. We refer to this as flavornutrient learning. The implication is that sucrose, when taken orally and subsequently metabolized, supports both forms of learning.

The motivational state of the subject appears to influence the contribution that each of these forms of learning makes to an observed preference (e.g., Fedorchak \& Bolles, 1987; Harris, Gorrissen, Bailey, \& Westbrook, 2000; Yiin, Ackroff, \& Sclafani, 2005). Fedorchak and Bolles showed that both saccharin and sucrose supported a preference in rats that were not hungry when tested. Inducing a state of hunger was found to enhance the magnitude of the preference for those trained with sucrose, but not for those trained with saccharin. Thus, flavortaste learning (supported by saccharin) is independent of the rat's motivational state. The results for sucrose may be interpreted as indicating that the preference based on flavortaste learning (evident when the animals are not hungry) is supplemented (or even replaced) by a preference based on flavor-nutrient learning when the animals are hungry (see also Harris et al., 2000). If we assume that, after training with sucrose as the US, hungry rats principally show the effects of flavor-nutrient learning at test, whereas sated rats show principally the effects of flavor-taste learning, then the results reported by Garcia-Burgos et al. (2013) can be interpreted as indicating that flavor-nutrient learning is susceptible to latent inhibition, whereas flavor-taste learning is not. This interpretation accords with the widely held view that flavor-nutrient learning is a form of expectancy learning that will obey the standard laws of conditioning, but that flavor-taste learning involves a different mechanism (e.g., one that produces a change in the hedonic properties of the flavor) that operates according to different laws (see, e.g., Campbell, Capaldi, Sheffer, \& Bradford, 1988; De Houwer, Thomas, \& Baeyens, 2001; Drucker, Ackroff, \& Sclafani, 1994; Pearce, 2002).

To test this interpretation of the results of Garcia-Burgos et al. (2013), it would be useful to have available procedures that allow for separate examinations of flavor-nutrient and flavor-taste learning. The former would be expected to show latent inhibition; the latter, not. A little information is already available, from experiments using somewhat unorthodox procedures. Weingarten and Kulikovsky (1989) reported results from a study investigating rats' response to sham-feeding, which they interpreted as supporting the proposal that preexposure to a flavor restricts the learning of an association between the flavor and the postingestive consequences of feeding. In contrast, no latent inhibition was found in a study by Galef and Durlach (1993), in which the training procedure involved allowing the subject rat to interact with another that had recently eaten food with a particular flavor. The enhanced flavor preference induced by this training (taken to reflect an association between the odor of the flavor and other cues produced by the demonstrator rat) was not prevented by preexposure to the flavor. Although these results are suggestive and consistent with the hypothesis under consideration, they come from complex procedures in which a range of factors would be operating, and alternative interpretations might be possible. Accordingly, in the experiments to be reported here, we have made use of the standard preferenceconditioning procedure and have attempted to isolate flavortaste and flavor-nutrient learning by making use of substances other than sucrose as the USs.

In our experiments, the USs were the monosaccharide fructose and the polysaccharide maltodextrin. There are reasons to think that a preference supported by the former is based principally on flavor-taste learning, whereas the latter principally promotes flavor-nutrient learning. Specifically, fructose has a sweet taste (there is good generalization between sucrose and fructose in rodents; Nissenbaum \& Sclafani, 1987), but it is not readily metabolized; consumed orally, it will support flavor-preference learning, but intragastric infusions are much less effective (Ackroff, Touzani, Peets, \& Sclafani, 2001; Sclafani et al., 1993). Maltodextrin, on the other hand, will support the conditioning of a flavor preference when it is presented by intragastric infusion (Ackroff \& Sclafani, 1994), but although its taste appears to be palatable (e.g., Dwyer, 2008), there is little evidence to indicate that its taste alone would engender a conditioned preference. Elizalde and Sclafani (1988) reported that ingested maltodextrin did not 
support preference learning when carbohydrate digestion was blocked by a drug treatment.

We acknowledge that the effects of these different USs may well be more complex than this. As we have just noted, intragastric fructose can support a degree of preference learning (albeit rather less strong than that produced by sucrose), implying that flavor-nutrient learning will occur to some extent with fructose. and Myers and Sclafani (2001) have demonstrated, using a taste reactivity test, that rats show positive responses to a flavor that has been associated with intragastric infusion of a sugar, implying that flavor-nutrient learning (like flavor-taste learning) may be capable of changing the hedonic response to a flavor. None the less, the strategy of comparing fructose with maltodextrin has been used with some success to address other issues in flavor-preference learning (e.g., Dwyer \& Quirk, 2008), and accordingly, we thought it worthwhile to look for latent inhibition in flavorpreference learning in rats trained with either fructose or maltodextrin as the US. Evidence of an effect with the latter but not the former would support the hypotheses (a) that different mechanisms underlie the preferences established by these USs, and (b) that the mechanism engaged by fructose (presumed to be flavor-taste learning) is not susceptible to latent inhibition.

\section{Experiment 1}

We created four groups of rats: Two were given preexposure to the almond flavor that was to be used as the CS, and the other groups received only water at this stage. During conditioning, one pair of groups consumed a mixture of almond and fructose, and the other pair a mixture of almond and maltodextrin. In a final test, all subjects were given access to two bottles, one containing the almond solution, and the other, unflavored water. In order to ensure that they would drink the fluids offered, the rats were water deprived throughout training. They had free access to food during preexposure and conditioning, but, given that a preference based on flavor-nutrient learning (such as maltodextrin is likely to produce) may only be fully evident in hungry animals, access to food was denied all subjects prior to the test.

\section{Method}

Subjects and apparatus The subjects were 32 naïve male Wistar rats (Janvier, France) with a mean body weight of $365 \mathrm{~g}$ at the start of the experiment. They were housed in individual home cages and kept in a colony room at the Biomedical Research Center of the University of Granada that was lit from 8:00 a.m. to 8:00 p.m. each day. Experimental procedures took place with the rats in their home cages and during the light period of the cycle. Inverted 50-ml plastic tubes equipped with stainless steel ball-bearing-tipped spouts were used to present fluids in these cages. Consumption was estimated by weighing the tubes before and after fluid presentation to the nearest $0.1 \mathrm{~g}$. Rats were maintained water deprived during preexposure and conditioning, but were both food and water deprived during the test. The solutions used were made up with tap water and consisted of $1 \%$ (vol/vol) almond essence (Shepcote Distributors Ltd, Yorkshire , UK) and a compound of $1 \%$ almond essence and $10 \%(\mathrm{wt} / \mathrm{vol})$ either maltodextrin (Maltodextrin white pure, Applichem, Darmstadt, Germany) or fructose (D[-]-Fructose, Panreac, Barcelona, Spain).

Procedure All of the experimental procedures were approved by the University of Granada Ethics Committee. To initiate the deprivation schedule, the water bottles were removed $24 \mathrm{~h}$ before the start of the experiment. The rats were then given three days to accommodate to a deprivation schedule, in which access to water was allowed twice a day for $30 \mathrm{~min}$, at 9:00 a.m. and 1:30 p.m. Rats were randomly allocated to two weight-matched groups - group Pre $(n=16)$ and group NPre $(n=16)$ - for the flavor exposure phase. This phase consisted of one single daily trial (at 9:00 a.m.) across eight days. Each trial consisted of $10 \mathrm{~min}$ access to $10 \mathrm{ml}$ of either almond (for animals in the Pre condition) or water (for animals in the NPre condition), followed by free access to water for $30 \mathrm{~min}$. An additional 30-min period of access to water was given each day at 1:30 p.m. After the exposure phase, rats were divided into four groups for the conditioning phase, matched on either average almond (groups Pre) or water (groups NPre) consumption during the preexposure phase, Pre/M $(n=8)$, NPre/M $(n=8)$, Pre/F $(n=8)$, and NPre/F $(n$ $=8)$.

Conditioning occurred over two days, with one trial each day during the morning session. In each trial, animals had $10 \mathrm{~min}$ access to $10 \mathrm{ml}$ of either almond + maltodextrin (groups M) or almond + fructose (groups F). During this 10min period, food was removed for all rats, in order to avoid any pairing of the flavor with the standard diet. After the last conditioning session, the rats had the second $30-\mathrm{min}$ access to water at 5:30 p.m. instead of 1:30 p.m., and food was then removed from the cages at 6:00 p.m. On the next day, the animals were tested while food deprived; the test consisted of $15 \mathrm{~min}$ access to two bottles at 10:00 a.m., one containing $20 \mathrm{ml}$ of the almond solution and the other $20 \mathrm{ml}$ of water. The positions of the bottles were counterbalanced across groups.

\section{Results and discussion}

All subjects drank readily during the preexposure phase. The mean consumptions (in grams) of almond over the course of this phase were 8.0 and 8.4 for groups Pre/M and Pre/F, respectively; the mean consumption of water was 8.4 for group 
NPre/M, and 8.3 for group NPre/F. An analysis of variance (ANOVA) with preexposure condition and reinforcer as variables yielded no significant main effects or interaction, largest $F(1,28)=1.62, p=.213, \eta_{\mathrm{p}}{ }^{2}=.05$.

The mean consumptions (in grams) of the almond + maltodextrin compound over the two conditioning trials were 8.7 for group Pre/M and 8.4 for group NPre/M. For groups given the almond + fructose compound, the means were 8.9 for group Pre/F and 6.7 for group NPre/F. Thus, we observed no difference in average consumption during conditioning between the preexposed and nonpreexposed rats when the reinforcer was maltodextrin, but preexposed animals conditioned with fructose drank more than the nonpreexposed ones. An ANOVA with Preexposure and Reinforcer as factors confirmed this impression, yielding a main effects of preexposure, $F(1,28)=6.48, p=.017, \eta_{\mathrm{p}}{ }^{2}=.19$, and a Preexposure $\times$ Reinforcer interaction, $F(1,28)=4.36, p=.046, \eta_{\mathrm{p}}{ }^{2}=.13$. The main effect of reinforcer was not significant, $F(1,28)=$ $2.66, p=.114, \eta_{\mathrm{p}}{ }^{2}=.09$. Post hoc comparison using Tukey's test showed that Pre/F rats drank significantly more than NPre/F rats. There was no difference between the preexposed and nonpreexposed animals when maltodextrin was the reinforcer.

Preference during the test was expressed as a ratio (consumption of almond/total consumption). Ratio scores are presented in Fig. 1 (left), where the groups are designated as $\mathrm{TTH}$, to indicate that they were thirsty $(\mathrm{T})$ during preexposure and conditioning, but also hungry $(\mathrm{H})$ for the test. It is evident that both NPre groups showed a greater preference for almond than did the Pre groups (i.e., latent inhibition was obtained with both reinforcers). An ANOVA with preexposure and reinforcer as the variables showed only a significant main effect of preexposure, $F(1,28)=4.64, p=.040, \eta_{\mathrm{p}}{ }^{2}=.10$; other $F \mathrm{~s}<$

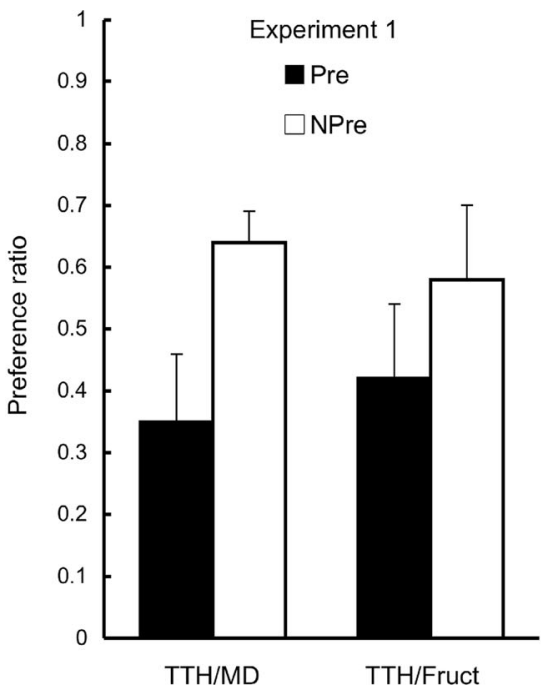

Fig. 1 Average preference ratios for almond over water for groups Pre (preexposed) and NPre (nonpreexposed) in Experiment 1 (left panel; groups TTH: $\mathrm{T}=$ thirsty, $\mathrm{H}=$ thirsty and hungry) and Experiment 2 (right
1. This difference is, in part, a consequence of the fact that the mean ratio scores for the Pre groups were less than .50. This should not be taken to imply that a learned aversion developed in these groups. With the stimuli and test procedures used here, rats given a choice between almond and water show a preference for water in the absence of any conditioning procedure (Garcia-Burgos et al., 2013, Exp. 3, reported a ratio score of about .40 in these circumstances). Thus, the rats in the Pre groups showed the slight aversion to almond that is found in untrained subjects, and this preference was reversed in the NPre groups. Note also that although rats in the Pre/F group drank more of the compound during conditioning than did those in the NPre/F group, their preference score was lower; the difference between these groups at test cannot be attributed to differences in consumption during conditioning.

The absolute scores for the consumption of water and the almond solution, on which the ratios were based, are presented in the top panel of Fig. 2. The pattern is the same for both reinforcers, with both NPre groups drinking less water than almond, but both Pre groups drinking less almond than water. There was substantial within-group variability in the absolute consumption scores, and an ANOVA on those for the consumption of almond, with preexposure condition and reinforcer as the variables, showed only a marginally significant main effect of preexposure, $F(1,28)=3.03, p=.09, \eta_{\mathrm{p}}{ }^{2}=.10$; other $F \mathrm{~S}<2$. The water scores showed no significant differences (all $\left.F_{\mathrm{S}}<1\right)$.

The results of this experiment indicate that latent inhibition, shown by a lesser preference for almond in animals exposed to the flavor before conditioning, occurs with both fructose and maltodextrin as the US. If we accept that each reinforcer produced just one of the two types of learning considered so far (flavor-taste learning for fructose and flavor-nutrient for

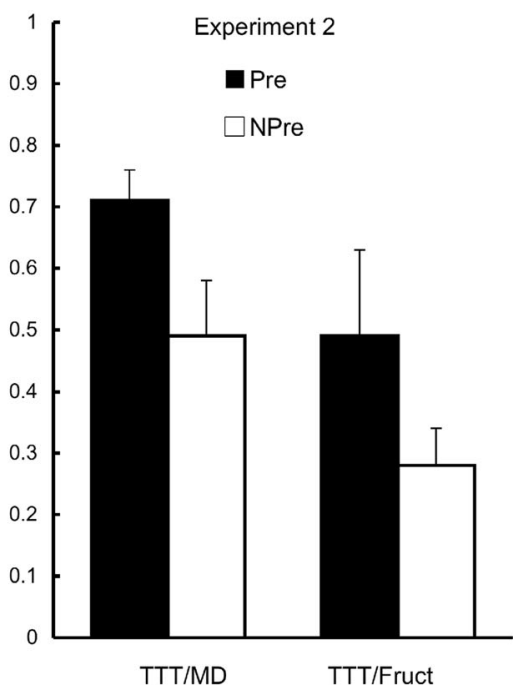

panel; groups TTT), for the reinforcers maltodextrin (MD) and fructose (Fruct). Error bars represent SEMs. 

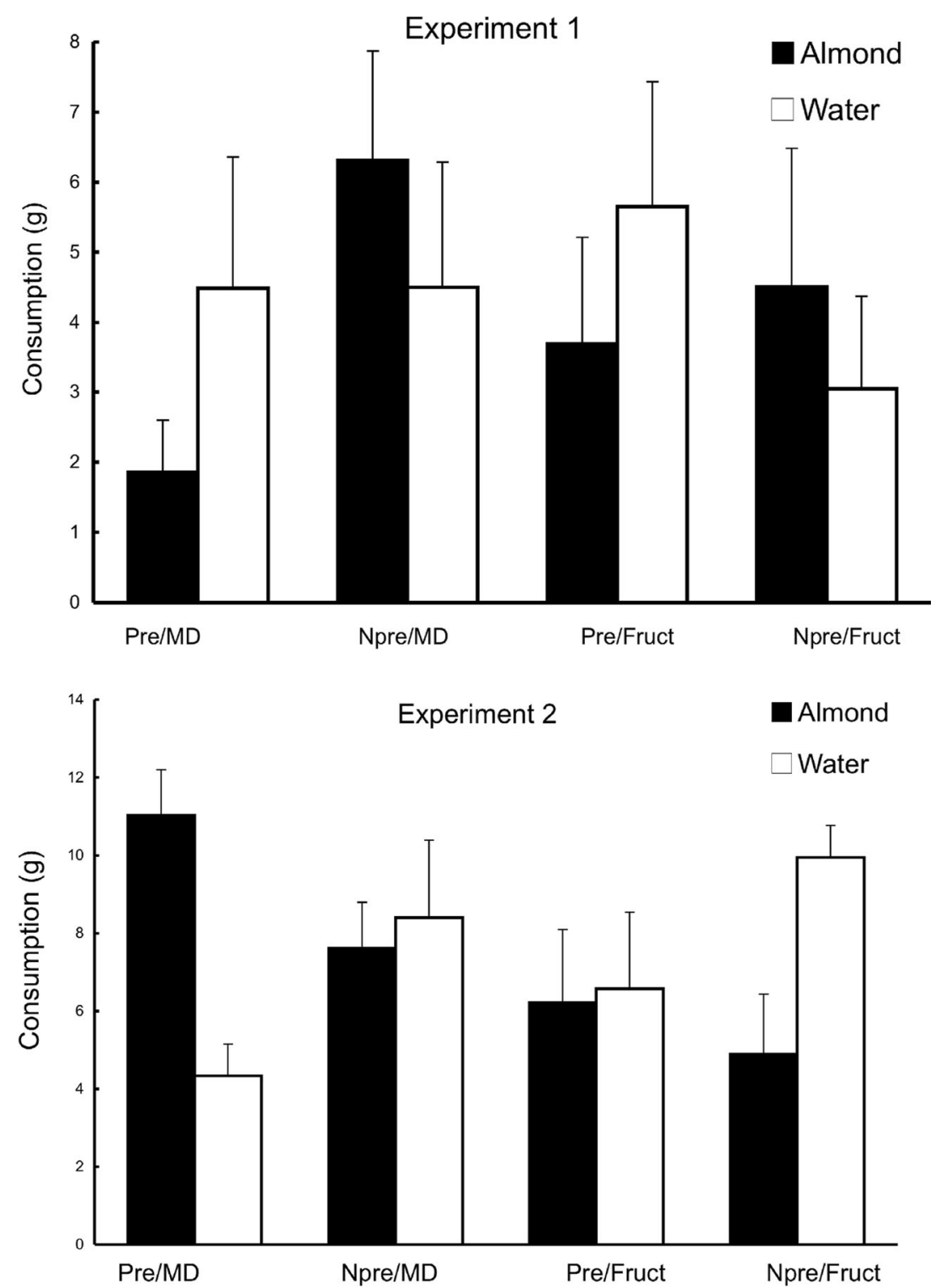

Fig. 2 Mean intakes (in grams) of almond and water for groups Pre (preexposed) and NPre (nonpreexposed) in Experiment 1 (top panel) and Experiment 2 (lower panel), for the reinforcers maltodextrin (MD) and fructose (Fruct). Error bars represent SEMs.

maltodextrin), we must conclude that the occurrence of latent inhibition does not depend of the content of learning. We found no support, therefore, for the hypothesis offered by Garcia-Burgos et al. (2013), on the basis of their experiments with sucrose as the US, that flavor-taste learning is immune to latent inhibition. The results of Garcia-Burgos et al., along with those reported so far, can be accommodated by the simpler hypothesis that making the animals hungry during the test increases its sensitivity, allowing latent inhibition to be obtained, whatever the properties of the reinforcer associated with the flavor.

Before discarding our original hypothesis, however, we should consider another possibility. Fructose is, after all, a sugar, and although its glycemic index is rather low, it is, nevertheless, capable of producing a degree of flavor-nutrient learning, and this might have been sufficient to generate a preference in our choice test procedure. If so, the results of this experiment could be taken to show, for fructose as for maltodextrin, that flavor-nutrient learning is susceptible to latent inhibition. The hypothesis that the rats needed to be hungry at test for the effects of flavor-nutrient learning to be seen suggests a possible test of this suggestion. If testing the rats when they are not hungry reveals a preference that depends chiefly on flavor-taste learning, then conditioning with fructose should still reveal a sizeable preference, but latent inhibition should be absent. Whether or not maltodextrin would generate a preference under these test conditions remains to be determined, and no simple prediction about the effects of preexposure was possible. But if a preference were observed, this should, according to our hypotheses, be a 
product of flavor-taste learning, and thus, like that for fructose, should be insensitive to latent inhibition.

\section{Experiment 2}

In this experiment, we again studied the four conditions used in Experiment 1 (preexposed or not preexposed to the CS, and maltodextrin or fructose as the US). This experiment differed only in that the rats were not deprived of food at any stage.

\section{Method}

The subjects were 32 male naïve Wistar rats (Janvier, France) with a mean body weight of $355 \mathrm{~g}$ at the start of the experiment. The housing, general maintenance, solutions, and apparatus were the same as we described for Experiment 1, with the exceptions mentioned below.

As in Experiment 1, the subjects in the Pre groups received eight sessions of preexposure to almond, and the NPre subjects received unflavored water, prior to conditioning with either the almond + fructose compound or the almond + maltodextrin compound, and then the choice test. Food was available throughout, except for the 30-min duration of each conditioning and test session. In our previous experiments investigating preferences in nonhungry rats (Garcia-Burgos et al., 2013), we gave two conditioning-test cycles (i.e., after the first test, the rats received two further sessions of conditioning, followed by another test), and this procedure was followed here.

\section{Results and discussion}

As in Experiment 1, the rats drank readily during preexposure, and we found no differences among the groups. The mean consumptions (in grams) of almond over the course of this phase were 8.2 for group Pre/M and 8.3 for group Pre/F. The mean consumptions of water were 8.4 for group NPre/M and 8.2 for group NPre/F. As we assessed by an ANOVA with preexposure and reinforcer as the variables, no significant effects were present, largest $F(1,28)=1.54, p=.225, \eta_{\mathrm{p}}{ }^{2}=.05$.

Although the rats drank most of the fluids offered on the conditioning trials, some small differences did emerge among the groups. The mean consumptions (in grams) of the almond + maltodextrin compound for the two conditioning cycles were 9.3 and 9.5 for group Pre/M, and 7.7 and 9.6 for group $\mathrm{NPre} / \mathrm{M}$. The equivalent means for consumption of the almond + fructose compound were 8.4 and 8.7 for group Pre/ F, and 6.4 and 7.7 for group NPre/F. An ANOVA with preexposure, reinforcer, and cycle as the variables yielded a significant main effect of reinforcer, $F(1,28)=15.80, p<$ $.001, \eta_{\mathrm{p}}{ }^{2}=.36$, confirming that maltodextrin was consumed more readily than fructose. We also found significant main effects of preexposure, $F(1,28)=13.12, p=.001, \eta_{\mathrm{p}}{ }^{2}=.32$, and cycle, $F(1,28)=33.58, p<.001, \eta_{\mathrm{p}}{ }^{2}=.54$, and a significant Cycle $\times$ Preexposure interaction, $F(1,28)=16.62, p<$ $.001, \eta_{\mathrm{p}}{ }^{2}=.37$. Analysis of the source of this interaction using post-hoc Tukey's tests showed that, whereas there were no differences in consumption over the two conditioning cycles in the preexposed animals, nonpreexposed rats drank less on the first than on the second cycle. We interpret this as indicating that nonpreexposed subjects showed a degree of neophobia on the first test cycle that had habituated by the second. The analysis produced no other significant interactions, largest $F(1,28)=1.31, p=.263, \eta_{\mathrm{p}}{ }^{2}=.04$.

Mean preference ratios pooled over the two tests for each group appear in Fig. 1 (right; the groups are now labeled TTT, for being only thirsty in all stages of the study). Scores were somewhat higher for maltodextrin than for fructose, and, in striking contrast to the results of Experiment 1, the preexposed groups showed a higher, rather than a lower, preference for almond, irrespective of the reinforcer. This reversal of the latent inhibition effect was quite unexpected. An ANOVA conducted on the average preference ratios, with preexposure and reinforcer as the variables, yielded significant main effects of preexposure, $F(1,28)=5.17, p=.031, \eta_{\mathrm{p}}{ }^{2}=.16$, and of reinforcer, $F(1,28)=5.48, p=.027, \eta_{\mathrm{p}}{ }^{2}=.16$; the interaction was not significant $(F<1)$.

Although the patterns of preference scores were the same for fructose and maltodextrin, inspection of the absolute consumption scores shows marked differences. The lower panel of Fig. 2 shows the group means for consumption of almond and water, pooled over both test trials. For maltodextrin, consumption levels of water and almond were approximately the same in subjects given no preexposure, but more almond than water was consumed by the preexposed animals. For fructose, on the other hand, the preexposed subjects were the ones to drink equal amounts, whereas the nonpreexposed subjects drank more water than almond. Rats conditioned with maltodextrin drank more almond than did those conditioned with fructose. An ANOVA on the scores for consumption of almond, with preexposure condition and reinforcer as the variables, showed a significant effect of the nature of the reinforcer, $F(1,28)=6.48, p=.017, \eta_{\mathrm{p}}{ }^{2}=.08$; the main effect of preexposure, $F(1,28)=2.57, p=.120$, and the interaction $(F<$ 1) were not significant. Water consumption, by contrast, was sensitive to the effects of preexposure, with preexposed subjects drinking less than nonpreexposed subjects. An ANOVA showed a significant effect of preexposure, $F(1,28)=6.03, p$ $=.021, \eta_{\mathrm{p}}{ }^{2}=.18$; the main effect of reinforcer, $F(1,28)=1.54$, $p=.225$, and the interaction $(F<1)$ were not significant.

The results of the absolute scores from a choice test can be difficult to interpret, given that they are not independent (if only because drinking from one bottle necessarily limits the opportunity to drink from the other). With this caveat, we offer the following as a possible interpretation of the results in the 
lower panel of Fig. 2. First, we suggest that one effect of preexposure is to allow habituation of neophobia to almond; subjects given preexposure will drink almond more readily, and thus drink less water than nonpreexposed subjects. Next, maltodextrin is more effective as a reinforcer than is fructose, so that more almond is drunk by subjects given the maltodextrin US than by those given the fructose US. The interaction of these two factors could have generated the results obtained. It will be noted, however, that this analysis has no place for a latent inhibition effect. The implications of this are taken up in the General Discussion.

\section{General discussion}

Latent inhibition is a powerful and well-documented effect, and any failure to observe retarded conditioning after extensive preexposure to the CS requires attention. The absence of latent inhibition in rats conditioned with sucrose as the US and tested when not hungry (Garcia-Burgos et al., 2013) prompted the hypothesis that a preference supported by flavor-taste learning is not susceptible to latent inhibition. In the present experiments, we attempted to test this hypothesis by making use of maltodextrin and fructose as the reinforcers and by testing preexposed and nonpreexposed subjects either in a state of hunger (Exp. 1) or when sated (Exp. 2). On the basis of the evidence presented in the Introduction, it was supposed that maltodextrin would engender flavor-nutrient learning, the effect of which would be evident in the preference shown by hungry animals, and that fructose would engender flavortaste learning, capable of producing a preference even in nonhungry animals.

The results of the experiments did not support these suppositions. We found, for subjects not given preexposure to the CS, that both USs were capable of generating a preference when the rats were hungry during the test, and when the rats were not hungry, neither produced a clear preference. If the proposed distinction between flavor-taste and flavor-nutrient learning is to be maintained, we must suppose that, with the stimuli and procedures used in our experiments, both USs are capable of producing flavor-nutrient learning, and that the effect of flavor-taste learning is weak for both reinforcers. This would impose limits on the strategy of using these two reinforcers to distinguish between two kinds of flavorpreference learning, suggesting that, at least under the conditions used in the present experiments, they may be functionally equivalent (for related results in the case of blocking, see González, Garcia-Burgos, \& Hall, 2014).

Nevertheless, the results for subjects given preexposure to the CS produced effects that are informative about the role of latent inhibition in flavor-preference conditioning. Experiment 1 , in which the animals were hungry during the test, produced evidence of latent inhibition with both reinforcers; that is, we observed an effect of preexposure in the expected direction that did not depend on the nature of the reinforcer. This result is consistent with the suggestion that flavor-nutrient learning occurs with both reinforcers and is susceptible to latent inhibition. In Experiment 2, however, when the animals were not hungry during the test, not only was no latent inhibition effect obtained, but unexpectedly, for both USs the preference for the conditioned flavor (as assessed by preference ratio scores) was greater in subjects given preexposure to the flavor (i.e., the reverse of latent inhibition was found).

To an extent, therefore, these findings confirm those of Garcia-Burgos et al. (2013), from their experiments using sucrose as the US, in that latent inhibition was evident in flavorpreference conditioning when the subjects were hungry during the test, but not when they were sated. An obvious interpretation of this pattern of results, at least at first sight, is that hunger promotes the expression of a preference (whatever the US), allowing conditioning to be seen clearly, and thus, the effect of preexposure to be observed. It might be argued that the absence of latent inhibition in the study by Garcia-Burgos et al. was merely a consequence of a low sensitivity to appetitive conditioning in nonhungry animals. But this cannot explain the results of the present Experiment 2, in which nonhungry rats showed a significant reversal of the latent inhibition effect. Other factors must be at work.

As we have noted, the results of Experiment 2 can be explained, in part, in terms of the rats' unconditioned response to the flavor. This factor could also have played a role in Experiment 1, but it seems reasonable to assume that the immediate response to the flavor of an ingested substance would be of more significance to a rat that is not in a state of hunger. The performance of those trained with fructose in Experiment 2 can be wholly explained in these terms. If we assume that fructose generated no substantial conditioned response in these test conditions, then the performance of the NPre group (a preference for water over almond) would be expected on the basis of a neophobic reaction to almond. Preexposure to almond, however, would allow habituation of neophobia, resulting in equal consumption of almond and water. The results for the maltodextrin groups are consistent with this analysis. These animals were more willing to drink almond, suggesting the operation of a conditioned preference. Such a preference could overcome neophobia in the NPre group, producing the result obtained, equal consumption of water and almond; and in the preexposed group, for which neophobia would not be factor, it could produce the marked preference for almond that was observed. This analysis does not require us to suppose that conditioning proceeded more readily in the Pre subjects. Critically, however, it does imply that the conditioning procedure was effective at producing a preference in the Pre group, and thus that latent inhibition was not effective in this condition. This is the outcome to be 
expected if flavor-taste learning is not subject to latent inhibition.

In summary, the use of maltodextrin and fructose as USs does not allow the clean separation of flavor-taste and flavornutrient learning that we had hoped for, but the manipulation of motivational state has a marked effect on latent inhibition. With both USs, preexposure retards the development of a conditioned preference for animals tested in a state of hunger, consistent with the conclusion that both generate flavor-nutrient learning that is susceptible to latent inhibition. When the subjects are not hungry during the test, there is an apparent reversal of latent inhibition. The source of this effect is complex and debatable, but we have offered a possible explanation that is consistent with the original proposal that flavor-taste learning is not susceptible to latent inhibition.

Author note This research was supported by Grant Number PSI201233552 (MINECO, Ministerio de Economía y Competitividad, Spain).

\section{References}

Ackroff, K., \& Sclafani, A. (1994). Flavor preferences conditioned by intragastric infusions of dilute Polycose solutions. Physiology \& Behavior, 55, 957-962. doi:10.1016/0031-9384(94)90086-8

Ackroff, K., Touzani, K., Peets, T. Y., \& Sclafani, A. (2001). Flavor preferences conditioned by intragastric fructose and glucose: Differences in reinforcement potency. Physiology \& Behavior, 72, 691-703.

Campbell, D. H., Capaldi, E. D., Sheffer, J. D., \& Bradford, J. P. (1988). An examination of the relationship between expectancy learning and preference conditioning. Learning and Motivation, 19, 162-182.

De Houwer, J., Thomas, S., \& Baeyens, F. (2001). Associative learning of likes and dislikes: A review of 25 years of research on human evaluative conditioning. Psychological Bulletin, 126, 853-869. doi:10. 1037/0033-2909.127.6.853

De la Casa, L. G., Márquez, R., \& Lubow, R. E. (2009). Super-latent inhibition of conditioned taste preference with a long retention interval. Learning and Motivation, 40, 329-342. doi:10.1016/j.1mot. 2009.03.001

Delamater, A. R. (2011). Partial reinforcement and latent inhibition effects on stimulus-outcome associations in flavor preference conditioning. Learning \& Behavior, 39, 259-270. doi:10.3758/s13420011-0026-6

Drucker, D. B., Ackroff, K., \& Sclafani, A. (1994). Nutrient-conditioned flavor preference and acceptance in rats: Effects of deprivation state and nonreinforcement. Physiology \& Behavior, 56, 701-707.
Dwyer, D. M. (2008). Microstructural analysis of conditioned and unconditioned responses to maltodextrin. Learning \& Behavior, 36, 149 158. doi:10.3758/LB.36.2.149

Dwyer, D. M., \& Quirk, R. H. (2008). Context conditional flavor preferences in the rat based on fructose and maltodextrin reinforcers. Journal of Experimental Psychology: Animal Behavior Processes, 34, 294-302. doi:10.1037/0097-7403.34.2.294

Elizalde, G., \& Sclafani, A. (1988). Starch-based conditioned preferences in rats: Influence of taste, calories, and CS-US delay. Appetite, 11, 179-200.

Fanselow, M. S., \& Birk, J. (1982). Flavor-flavor associations induce hedonic shifts in taste preference. Animal Learning \& Behavior, 10, 223-228.

Fedorchak, P. M., \& Bolles, R. C. (1987). Hunger enhances the expression of calorie- but not taste-mediated conditioned flavor preferences. Journal of Experimental Psychology: Animal Behavior Processes, 13, 73-79. doi:10.1037/0097-7403.13.1.73

Galef, B. G., \& Durlach, P. J. (1993). Absence of blocking, overshadowing, and latent inhibition in social enhancement of food preferences. Animal Learning \& Behavior, 21, 214-220.

Garcia-Burgos, D., González, F., \& Hall, G. (2013). Motivational control of latent inhibition in flavor preference. Behavioural Processes, 98, 9-17.

González, F., Garcia-Burgos, D., \& Hall, G. (2014). Analysis of blocking of flavor-preference conditioning based on nutrients and palatable tastes in rats. Appetite, 80, 161-167.

Harris, J. A., Gorrissen, M. C., Bailey, G. K., \& Westbrook, R. F. (2000). Motivational state regulates the content of learned flavor preferences. Journal of Experimental Psychology: Animal Behavior Processes, 26, 15-30. doi:10.1037/0097-7403.26.1.15

Lubow, R. E. (1989). Latent inhibition and conditioned attention theory. New York, NY: Cambridge University Press.

Myers, K. P., \& Sclafani, A. (2001). Conditioned enhancement of flavor evaluation reinforced by intrgastric glucose: II. Taste reactivity analysis. Physiology \& Behavior, 74, 495-505.

Nissenbaum, J. W., \& Sclafani, A. (1987). Qualitative differences in polysaccharide and sugar tastes in the rat: A two-carbohydrate taste model. Neuroscience \& Biobehavioral Reviews, 11, 187-196.

Pearce, J. M. (2002). Evaluation and development of a connectionist theory of configural learning. Animal Learning \& Behavior, 30, 73-95. doi:10.3758/BF03192911

Sclafani, A., Cardieri, C., Tucker, K., Blusk, D., \& Ackroff, K. (1993). Intragastric glucose but not fructose conditions robust flavor preferences in rats. American Journal of Physiology. Regulatory, Integrative and Comparative Physiology, 265, R320-R325.

Weingarten, H. P., \& Kulikovsky, O. T. (1989). Taste-to-postingestive consequence conditioning: Is the rise in sham feeding with repeated experience a learning phenomenon? Physiology \& Behavior, 45, 471-476.

Yiin, Y. M., Ackroff, K., \& Sclafani, A. (2005). Food deprivation enhances the expression but not acquisition of flavor acceptance conditioning in rats. Appetite, 45, 152-160. 